\title{
Large Mode Area Photonic Crystal Fiber with Low Dispersion and Confinement Loss for Terahertz Wave Guiding
}

\author{
Chao Liu ${ }^{1 *}$, Liying Wang ${ }^{1}$, Jianwei Wang ${ }^{1}$, Sinuo An ${ }^{1}$, Famei Wang ${ }^{2}$, Xianli $\mathrm{Li}^{1 *}$, Wei Liu ${ }^{1}$, Weiquan $\mathrm{Su}^{1}$, \\ Qiang Liu ${ }^{1}$, Haiwei $\mathrm{Mu}^{1}$, Tao $\mathrm{Sun}^{3}$, Paul K. Chu ${ }^{4}$ \\ ${ }^{1}$ School of Electronics Science, Northeast Petroleum University, Daqing 163318, P. R. China \\ ${ }^{2}$ School of Materials Science and Chemical Engineering, Harbin Engineering University, Harbin 150001, P. R. China \\ ${ }^{3}$ Media Lab, Massachusetts Institute of Technology, Cambridge, MA 02139, USA \\ ${ }^{4}$ Department of Physics and Department of Materials Science and Engineering, City University of Hong Kong, Tat Chee Avenue, \\ Kowloon, Hong Kong, China \\ Email: msm-liu@126.com,1x17158@163.com
}

\begin{abstract}
A photonic crystal fiber (PCF) made of the cyclic-olefin copolymer (COC) with low dispersion and confinement loss, large-mode-area, and single-mode transmission for terahertz wave guiding is described. The characteristics of the PCF in the terahertz range are simulated and analyzed by the full-vector finite element method (FEM). The effects of the structural parameters on the performance of the terahertz PCF are also investigated. The effective mode field area of the PCF is as large as $1.22084 \times 10^{7} \mu \mathrm{m}^{2}$ at a wavelength of $1,000 \mu \mathrm{m}$ and a flat dispersion of $0.07669 \pm 0.33258 \mathrm{ps} / \mathrm{nm} / \mathrm{km}$ is obtained. A much lower confinement loss of $6.0253 \times 10^{-16} \mathrm{~dB} / \mathrm{m}$ is achieved. The single mode transmission over the entire terahertz wave band is described.
\end{abstract}

Keywords: photonic crystal fiber, low confinement loss, large mode area, terahertz

\section{Introduction}

The terahertz $(\mathrm{THz})$ frequency ranges from $0.1 \mathrm{THz}$ to $10 \mathrm{THz}$ between microwave and infrared light, corresponding to the wavelength range of $30 \mu \mathrm{m}$ to $3 \mathrm{~mm}^{[1]}$. Terahertz waves are promising in $\mathrm{THz}$ time domain spectroscopy ${ }^{[2]}$, THz imaging $^{[3]}$, security check ${ }^{[4]}$, astronomy ${ }^{[5]}$, and biomedical science ${ }^{[6]}$. However, one of the challenges confronting terahertz wave transmission is strong transmission $\operatorname{loss}^{[7]}$ and so it is crucial to design a high-efficiency and low-loss THz waveguide. Up to date, many $\mathrm{THz}$ waveguides have been proposed and $\mathrm{THz}$ metal waveguide have a low absorption loss during propagation of the THz wave propagates ${ }^{[8,9]}$.

Recently, the photonic crystal fiber (PCF) has attracted much attention as a promising terahertz waveguide boasting high design flexibility. It is a special type with periodically arranged air holes spanning the length of the fiber ${ }^{[10-12]}$. PCF possesses excellent features that can not be realized by conventional fibers, for example, high birefringence ${ }^{[13]}$, endless single-mode transmission ${ }^{[14]}$, adjustable dispersion ${ }^{[15]}$, and large effective mode area ${ }^{[16]}$. Nielsen et al. designed a terahertz PCF with Topas ${ }^{[17]}$ and porous-core band gap terahertz $\mathrm{PCF}^{[18]}$ and the losses observed from the two fibers were approximately $0.1 \mathrm{~dB} / \mathrm{cm}$ and $0.25 \mathrm{~dB} / \mathrm{cm}$. R. Pobre et al. studied the single mode characteristics of the Teflon-based PCF and found that the factor affecting the single-mode transmission range was the size of the cladding air holes ${ }^{[19]}$. S. Lou et al. designed a hexagonal lattice structure for the polyethylene PCF in THz frequencies and dispersion was close to zero when the frequency was higher than $1 \mathrm{THz}^{[20]}$. Owing to the superior characteristics of PCF in terahertz guiding, much effort has been devoted to terahertz PCF waveguides. The large mode area, low confinement loss, and flat dispersion are the objectives being pursued. Recently, it has been shown that PCF with polymeric materials can lower the transmission loss of $\mathrm{THz}$ wave. In fact, polymeric materials tend to be cheap and are widely available compared to other dielectrics. For instance, high-density polyethylene (HDPE) and polytetrafluoroethylene (Teflon) have very low transmission losses in the $\mathrm{THz}_{\text {band }}{ }^{[21-23]}$ and COC has a small absorption coefficient ${ }^{[24]}$.

Herein, we describe an octagonal density circle THz-PCF structure with low dispersion, low confinement loss, large mode area, and single-mode transmission over the entire range between $30 \mu \mathrm{m}$ and $3 \mathrm{~mm}$. The THz-PCF has excellent characteristics and large potential in optical waveguides. 


\section{Numerical modeling}

In this work, a full vectorial finite element method (FEM) was used as the calculation and analytical tool based on the COMSOL Multiphysics software. It has been demonstrated that FEM is applicable to the calculation and analysis of various complex model structures ${ }^{[25]}$. Figure 1 shows the schematic diagram of the structure. The blue area represents the base materials of the cyclic olefin copolymer with very low light absorption loss and a refractive index of 1.5258 in the $\mathrm{THz}$ band $^{[26]}$. The pink area is a perfectly matched layer (PML) for absorption of radiation ${ }^{[27]}$. Dense air holes are periodically arranged on the base materials to reduce the refractive index of the cladding. The outer four layers are octagonal and the innermost layer is circular. For the arrangement of air holes, octagonal PCF has many advantages compared with hexagonal PCF. For example, in the single-mode region, the wavelength range is wider, and the distribution of the mode field is closer to the circle and the limiting loss is smaller ${ }^{[28]}$. In addition, the innermost air holes are arranged in a circular manner, which is to concentrate more energy on the fiber core and reduce transmission loss. The outer air holes and innermost air hole diameter are, respectively, denoted by $d_{1}, d_{2}$ and the spacing between layers is denoted as $\Lambda$. The distribution of dense circles of the cladding is to better confine light to the core propagation. In order to monitor changes in the distribution of the mode field, the structural parameters are set to be $\Lambda=400 \mu \mathrm{m}, d_{1}=100 \mu \mathrm{m}, d_{2}=60 \mu \mathrm{m}$. The fundamental mode distributions in each frequency band are shown in Figure 2 which shows that light is well confined to propagate in the core and the pores have better light confinement capability as the frequency is increased.

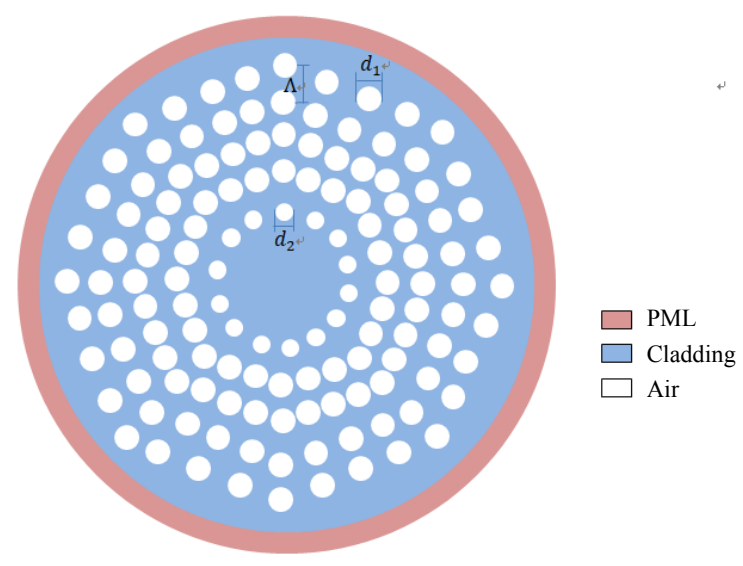

Figure 1. Cross section of the THz-PCF structure

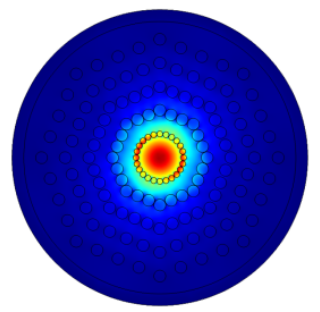

(a) $0.3 \mathrm{THz}$

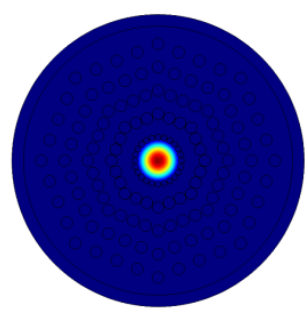

(d) $3.0 \mathrm{THz}$

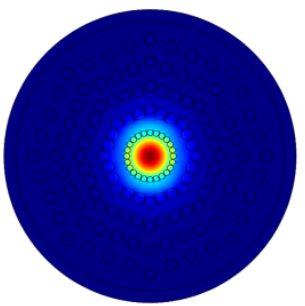

(b) $0.4 \mathrm{THz}$

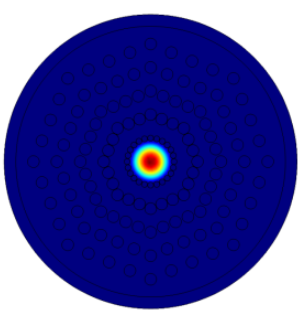

(e) $7.0 \mathrm{THz}$

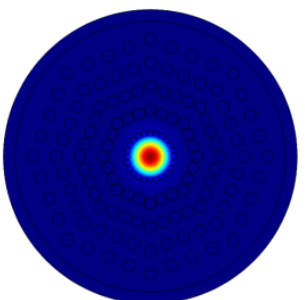

(c) $1.0 \mathrm{THz}$

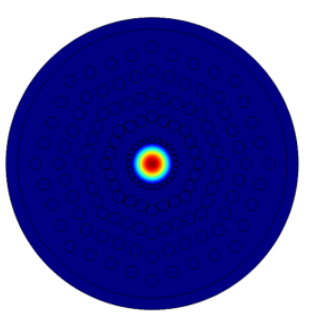

(f) $10.0 \mathrm{THz}$

Figure 2. Electric field distribution at different frequencies

The structural parameters of the PCF terahertz waveguide have large influence on the propagation characteristics, 
including the single-mode transmission range, effective mode area, dispersion, and transmission loss. These parameters can be optimized by adjusting the structural parameters of the PCF. In general, endless single-mode transmission is crucial to optical fibers because it prevents signal distortion and power loss caused by the transitions between modes. An important indicator of whether a fiber can perform single-mode transmission is the normalized frequency $V$. For the PCF, the normalized frequency $V_{P C F}$ can be expressed as [29]:

$$
V_{P C F}=\frac{2 \pi p}{\lambda}\left(n_{c o}{ }^{2}-n_{e f f}{ }^{2}\right)^{1 / 2}
$$

where $\rho$ is the equivalent core radius, $\lambda$ is the vacuum wavelength, $n_{c o}$ is the refractive index of the core, and $n_{e f f}$ represents the refractive index of the cladding. Because the fiber cross-section is covered with regularly arranged air holes, the fiber cross-section has an equivalent refractive index distribution. This equivalent refractive index is also the equivalent refractive index of the cladding. Since there is no air hole in the core of the PCF, the refractive index of the core is equal to the refractive index of the base material. The normalized frequency is an important factor to realize single-mode transmission in the PCF. In general, the PCF can achieve single-mode transmission when $V_{P C F}$ is less than $2.405^{[30]}$.

The effective mode field area is another critical parameter and it is often used to change the nonlinearity of PCF. A large mode field area is expected because it not only performs light transmission, but also reduces the nonlinearity of the PCF. The effective mode field area can be obtained by the following formula ${ }^{[31]}$ :

$$
A_{e f f}=\frac{\left(\iint|E|^{2} d x d y\right)^{2}}{\iint|E|^{4} d x d y}\left(\mu m^{2}\right)
$$

where $E$ is the amplitude of the electric field of the basic model. The dispersion of the PCF consists of materials dispersion and waveguide dispersion. In this work, only waveguide dispersion is considered because materials dispersion in the cycloolefin copolymer is close to zero and almost negligible. Dispersion is one of the primary limiting factors of the transmission distance of optical fiber communication systems and hence, flat low dispersion is desirable. Waveguide dispersion of the PCF can be expressed as ${ }^{[32]}$ :

$$
D=-\frac{\lambda}{c} \frac{\partial^{2}\left|\operatorname{Re}\left(n_{e f f}\right)\right|}{\partial \lambda^{2}}
$$

where $c$ is the light speed in vacuum and $\operatorname{Re}\left(n_{e f f}\right)$ is real part of effective refractive index.

The confinement loss is an important parameter for the fiber THz waveguides. The confinement loss can be calculated from the imaginary part of the effective mode index of refraction obtained from the added PML boundary conditions as expressed in the following ${ }^{[33]}$ :

$$
L_{c o n}=8.686 k_{0} \operatorname{Im}\left(n_{\text {eff }}\right)
$$

where $k_{0}$ is the value of $2 \pi / \lambda, \operatorname{Im}\left(n_{\text {eff }}\right)$ is the imaginary part of the effective mode index.

\section{Results and discussion}

Figure 3 shows the numerical results of $V_{P C F}$ in the proposed PCFs at different frequencies. It is observed from Figure 3 (a) and Figure 3(b) that the values of $V_{P C F}$ of the PCFs are less than 2.405 regardless of changing $d_{1}, d_{2}$, and $\Lambda$. This result indicates that the PCF in this study can perform infinite single mode transmission in the THz band. The single-mode transmission range of this model is larger than those results in Ref. ${ }^{[34]}$. 


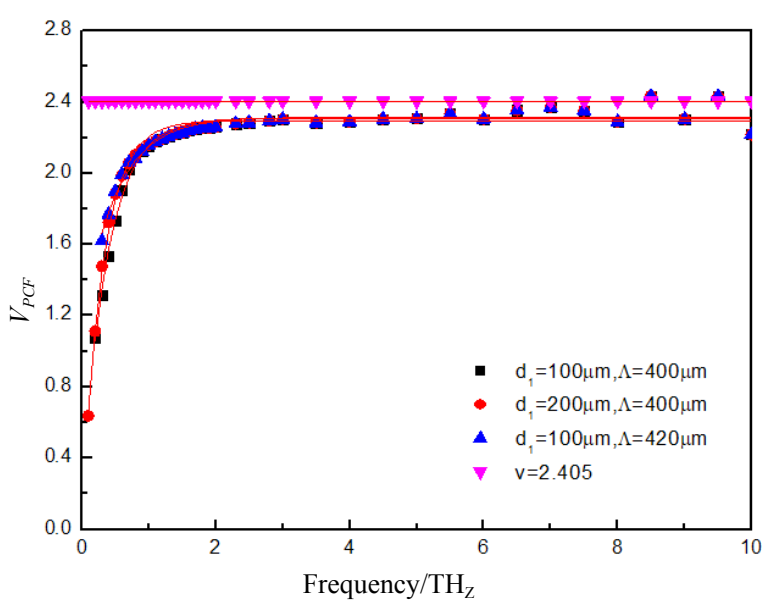

(a)

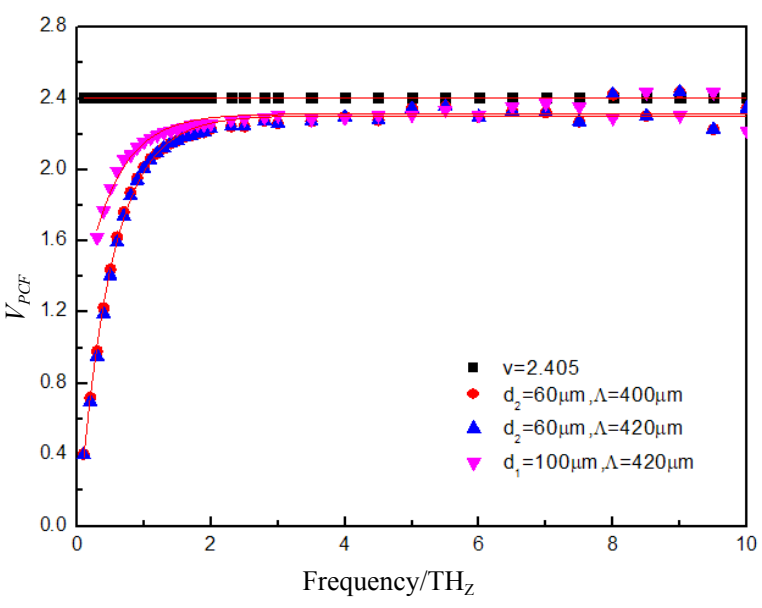

(b)

Figure 3. Dependency of $V_{P C F}$ on frequencies for different structural parameters

Figure 4 illustrates the dependence of the effective refractive index of the PCF on frequencies. The real part of the effective refractive index increase initially with frequencies and then reaches a stable value corresponding to the refractive index of the base materials. The imaginary part of the effective mode refractive index decreases with frequency and stabilizes. This phenomenon is attributed to diffusion of the mode field to the cladding at the low frequency band. The restriction power of the cladding increases and energy is confined to the core as the frequency increases.

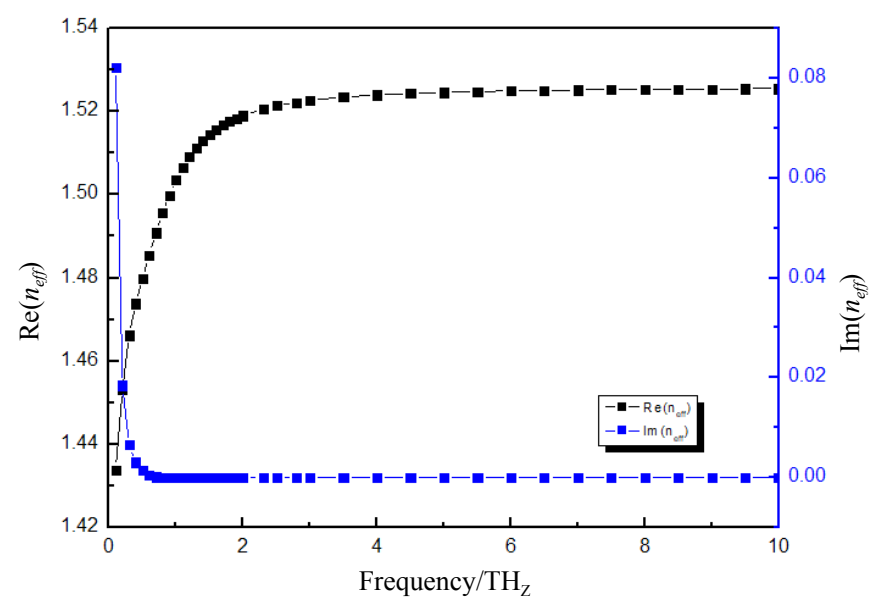

Figure 4. Dependences of the effective refractive index of the PCF on frequencies

Figure 5 shows the variations of the effective mode field areas for different structural parameters of the PCF. Figure 5(a) and 5(b) show that the structural parameters have little effects on the effective mode field area when the wavelength is less than $375 \mu \mathrm{m}$. However, influences of the structural parameters on the effective mode field area are more obvious when the wavelength is larger than $375 \mu \mathrm{m}$. It can be seen from the results shown in Figure 5 that the binding light beams ability of the THz wave is weakened when the wavelengths exceed $400 \mu \mathrm{m}$, which causes more energy to diffuse into the cladding, thus leading to be a sharp increase in the effective mode field area. The maximum effective mode field area at a wavelength of $1,000 \mu \mathrm{m}$ is $1.41868 \times 10^{7} \mu \mathrm{m}^{2}$ for $d_{1}=100 \mu \mathrm{m}, d_{2}=100 \mu \mathrm{m}$, and $\Lambda=400 \mu \mathrm{m}$, as shown in Figure 5(a). In addition, Figure 5(b) shows that $d_{2}$ has a greater influence on the effective mode field area. When $d_{1}, d_{2}$, and $\Lambda$ are $100 \mu \mathrm{m}$, $100 \mu \mathrm{m}$ and $420 \mu \mathrm{m}$, respectively, the effective mode field area reaches $1.22084 \times 10^{7} \mu \mathrm{m}^{2}$ at $1,000 \mu \mathrm{m}$, and the values are much larger than those in Refs. [35] and [36]. 


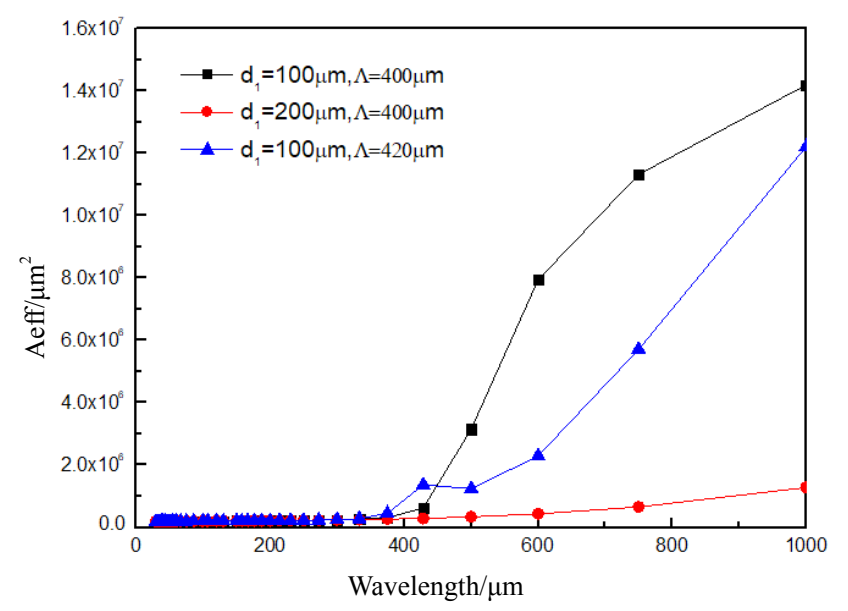

(a)

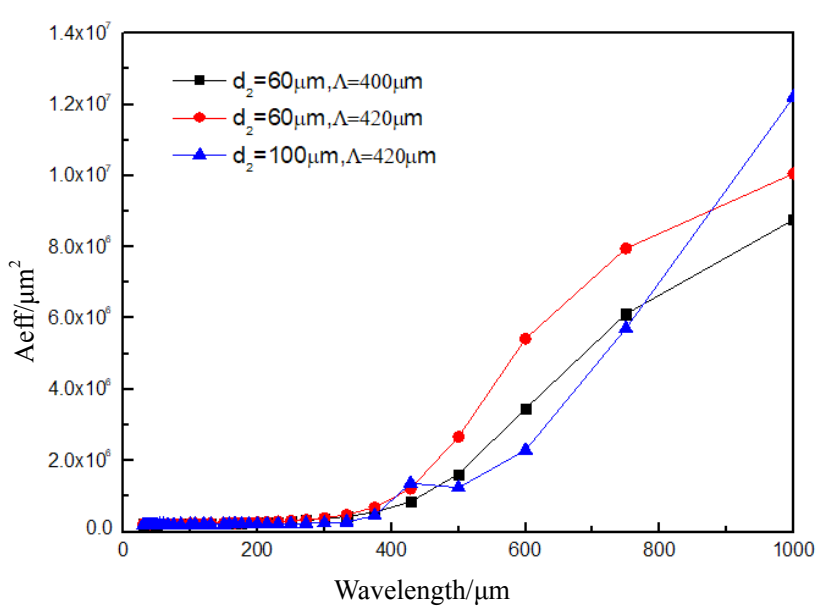

(b)

Figure 5. Variations of effective mode field areas for various parameters of the PCF

Figure 6 shows the dispersion characteristics of the PCF with different structural parameters in the terahertz region. As shown in Figure 6(a), the black curve is relatively flat indicating that dispersion is small in this wavelength range. Figure 6(b) shows that $d_{2}$ has a larger effect on dispersion. The ultra-flat dispersion of $0.07669 \pm 0.33258 \mathrm{ps} / \mathrm{nm} / \mathrm{km}$ is obtained in the entire THz band for $d_{1}=100 \mu \mathrm{m}, d_{2}=60 \mu \mathrm{m}$, and $\Lambda=400 \mu \mathrm{m}$. The dispersion obtained from this structure is flatter and smaller than the results in Refs. [37] and [38].

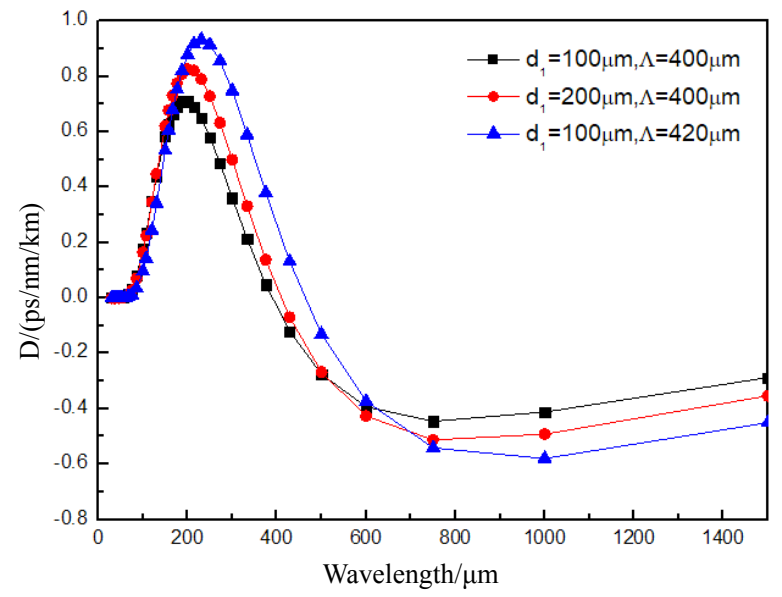

(a)

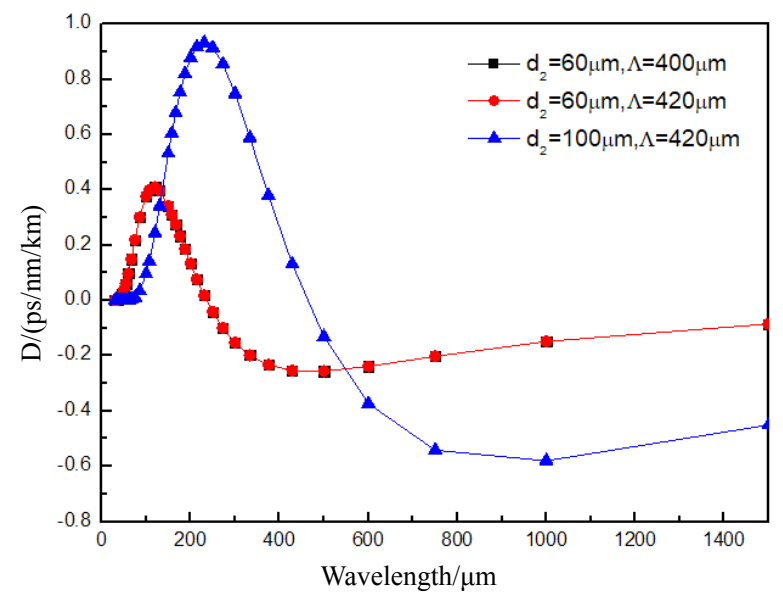

(b)

Figure 6. Dispersion characteristics of the PCF for different structure parameters in terahertz region

The confinement loss characteristics of the THz-PCF are shown in Figure 7. The structural parameters are $d_{1}=200$ $\mu \mathrm{m}, d_{2}=100 \mu \mathrm{m}$, and $\Lambda=400 \mu \mathrm{m}$. The confinement loss increases with increasing wavelengths when the wavelength is larger than a specific value. The confinement loss is low between $30 \mu \mathrm{m}$ and $334 \mu \mathrm{m}$ and a minimum confinement loss of $6.0253 \times 10^{-16} \mathrm{~dB} / \mathrm{m}$ can be achieved at $214 \mu \mathrm{m}$. The obtained confinement loss is smaller than those described in Refs. [34] and [39]. Obviously, it can be known from Figure 7 that $d_{1}$ and $d_{2}$ have higher effect on confinement loss than air holes spacing. 


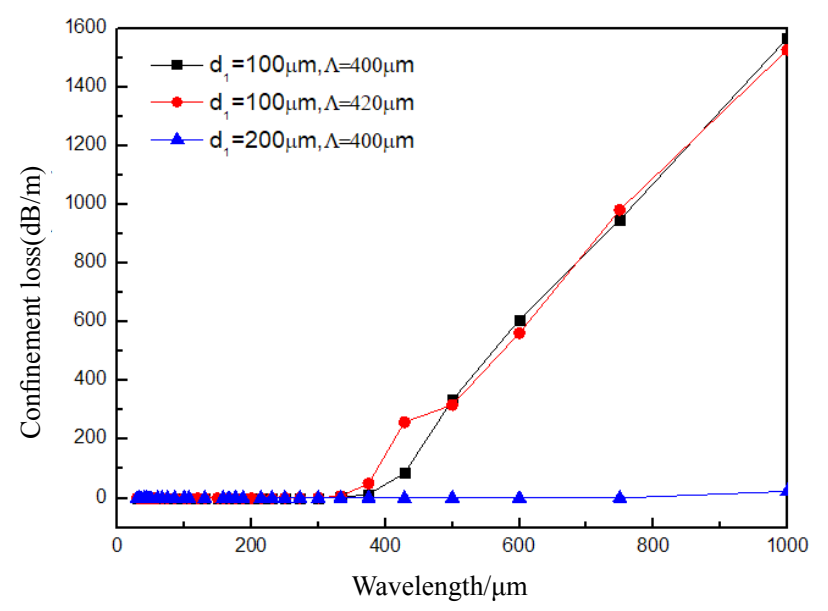

(a)

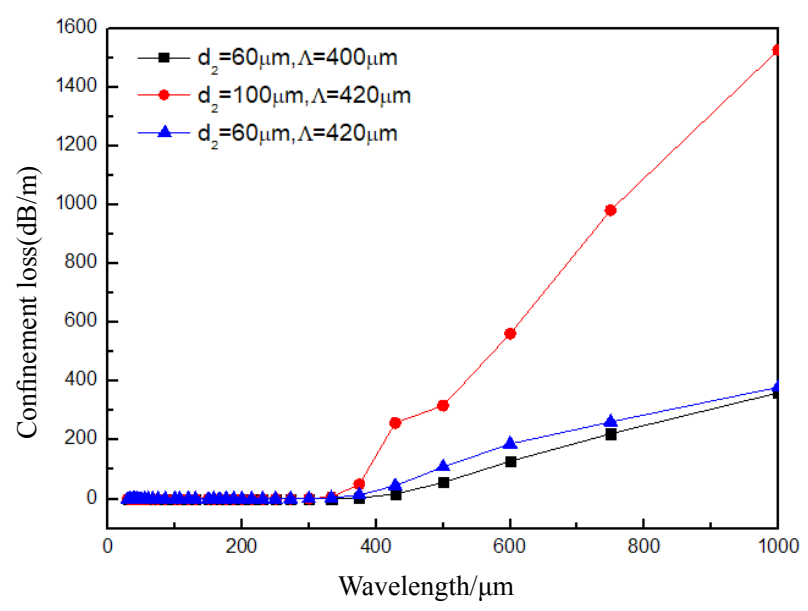

(b)

Figure 7. Confinement losses characteristics of the THz-PCF under different structural parameters

\section{Conclusion}

A THz-PCF made of COC boasting low dispersion and confinement loss, large-mode-area, and single mode transmission over the entire $\mathrm{THz}$ range is described. Our results show that the effective mode field area is as large as 1.22084 $\times 10^{7} \mu \mathrm{m}^{2}$ at $1,000 \mu \mathrm{m}$ together with an ultra-flat dispersion of $0.07669 \pm 0.33258 \mathrm{ps} / \mathrm{nm} / \mathrm{km}$. A confinement loss of 6.0253 $\times 10^{-16} \mathrm{~dB} / \mathrm{m}$ is achieved at $214 \mu \mathrm{m}$. Single-mode transmission is realized over the entire THz wave band and this THz-PCF has superior characteristics. The PCF terahertz wave guiding proposed in this work can be used in wireless communication and long-distance optical signal transmission.

\section{Acknowledgments}

This work was jointly supported by the National Natural Science Foundation of China [grant numbers 51474069, 41472126]; China Postdoctoral Science Foundation funded project [grant number 2016M591510]; Natural Science Foundation of Heilongjiang Province [grant number E2016007]; and Hong Kong Innovation and Technology Fund (ITF) ITS/452/17FP [CityU 9440179].

\section{References}

[1] Kim S, Kee CS, Lee JM. Single-mode Condition and Dispersion of Terahertz Photonic Crystal Fiber. Journal of the Optical Society of Korea. 2007; 11(3): 97-100.

[2] Tomasino A, Parisi A, Stivala S, et al. Wideband THz Time Domain Spectroscopy based on Optical Rectification and Electro-Optic Sampling. Scientific Reports. 2013; 3(10): 3116.

[3] Sun HQ, Zhao GZ, Tian Y, et al. Transverse mode distribution of THz Wave by THz imaging technology. Chinese Journal of Lasers. 2006; 33(9): 1225-1229.

[4] Ren L. Application of Terahertz Technology in Railway Security Checks. Electronic Test. 2014; 118-120.

[5] Shi SC. Development of superconducting mixers and detectors for THz astronomy. Microwave Conference. 2017; 55(1): 120-126.

[6] Hunt NI. Terahertz Biomedical Science and Technology. Journal of Biomedical Optics. 2014; 19(12): 129901-129901.

[7] Li S, Liu H, Huang N, et al. Broadband high birefringence and low dispersion terahertz photonic crystal fiber. Journal of Optics. 2014; 16(10): 105102.

[8] Mendis R. Nature of subpicsecond terahertz pulse propagation in practical dielectric-filled parallel-plate waveguides. Optics Letter. 2006; 31(17): 2643.

[9] Liu JM, Zhang M, Su H. Dielectric-gap-metal waveguides for THz low-loss propagating wave with subwavelength mode width. Applied Optics \& Photonics China. 2015; 9672: 96720J.

[10] Russell P. Photonic crystal fibers. Science. 2007; 24(12): 358-362.

[11] Cordeiro CMB, Franco MAR, Chesini G. Microstructured-core optical fibre for evanescent sensing applications. Opt. Express. 2006; 14(26): 13056-13066.

[12] Arif MFH, Biddut MJH. Enhancement of relative sensitivity of photonic crystal fiber with high birefringence and low 
confinement loss. Optik-International Journal for Light and Electron Optics. 2016; 131.

[13] Sun Y-S, Chau Y-F, Yeh H-H, et al. High birefringence photonic crystal fiber with a complex unit cell of asymmetric elliptical air-hole cladding. Applied Optics. 2007; 46(22): 5276-81.

[14] Knight J C, Birks T A, Russell P S J, et al. All silica single-mode optical fiber with photonic crystal cladding. Optics Letters. 1996; 21(19): 1547-1549.

[15] Saitoh K, Koshiba M, Hasegawa T, et al. Chromatic dispersion control in photonic crystal fibers: application to ultraflattened dispersion. Optics Express. 2003; 11(8): 843.

[16] Abdelaziz I, Ademgil H, Abdelmalek F, et al. Design of a large effective mode area photonic crystal fiber with modified rings. Optics Communications. 2010; 283(24): 5218-5223.

[17] Nielsen K, Rasmussen H K, Adam A J L, et al. Bendable, low-loss topas fibers for the terahertz frequency range. Optics Express. 2009; 17(10): 8592-601.

[18] Cho M, Kim J, Park H, et al. Highly birefringent terahertz polarization maintaining plastic photonic crystal fibers. Optics Express. 2008; 16(1): 7-12.

[19] Pobre R, Quema A, Murakami H. Single mode characteristics of Teflon photonic crystal fiber (PCF) in the subterahertz (THz) region. Conference on Lasers \& Electro-optics. 2005; 639-640.

[20] Lou S, Fang H, Jian S. Polyethylene photonic crystal fibers as terahertz waveguides. 2005.

[21] Argyros A, Leon-Saval S G, Pla J, et al. Anti-resonant reflection and inhibited coupling in hollow-core square lattice optical fibres. Optics express. 2008; 16(8): 5642-5648.

[22] Lai C H, You B, Lu J Y. Modal characteristics of anti-resonant reflecting pipe waveguides for terahertz waveguiding. Optics express. 2010; 18(1): 309-322.

[23] Wu D S, Argyros A, Leon-Saval S G. Reducing the size of hollow terahertz waveguides. Journal of Lightwave Technology. 2011; 29(1): 97-103.

[24] Ung B, Mazhorova A, Dupuis A. Polymer microstructured optical fibers for terahertz wave guiding. Optics Express. 2011; 19(26): 848-61.

[25] Ademgil H. Highly sensitive octagonal photonic crystal fiber based sensor. Optik-International Journal for Light and Electron Optics. 2014; 125(20): 6274-6278.

[26] Chen Q, Zhang YX, He XY, et al. Design and fabrication of Cyclic-olefin Copolymer based terahertz hollow-core photonic crystal fiber. International Conference on Infrared. 2013; 12(1): 1-2.

[27] Selleri S, Vincetti L., Cucinotta A, et al. Complex FEM modal solver of optical waveguides with PML boundary conditions. Opt. Quant. Electron. 2001; 33: 359-371.

[28] Chiang J S, Wu T L. Analysis of propagation characteristics for an octagonal photonic crystal fiber (O-PCF). Optics Communications. 2006; 258(2): 170-176.

[29] Ani, A. B., Faisal, M. Ultra-flattened broadband dispersion compensating photonic crystal fiber with ultra-low confinement loss. International Conference on Electrical \& Computer Engineering. 2017.

[30] Nagel S, Macchesney J, Walker K. An Overview of the Modified Chemical Vapor Deposition (MCVD) Process and Performance. IEEE Journal of Quantum Electronics. 2003; 18(4): 459-476.

[31] De, M., Gangwar, R. K., Singh, V. K. Designing of highly birefringence, dispersion shifted decagonal photonic crystal fiber with low confinement loss. Photonics and Nanostructures-Fundamentals and Applications. 2017; $26: 15-23$.

[32] Saha, R., Hossain, M. M., Rahaman, M. E., Mondal, H. S. Design and analysis of high birefringence and nonlinearity with small confinement loss photonic crystal fiber. Frontiers of Optoelectronics. 2019; 11(42): 315-418.

[33] Exian, L., Wei, T., Bei, Y., et al. Broadband ultra-flattened dispersion, ultra-low confinement loss and large effective mode area in an octagonal photonic quasi-crystal fiber. Journal of the Optical Society of America A. 2018; 35(3): 431.

[34] Pobre R, Quema A, Murakami H, et al. Single mode characteristics of teflon photonic crystal fiber (PCF) in the subterahertz (THz) region. Conference on Lasers \& Electro-optics. 2005; 639-640.

[35] Razzak SMA, Namihira Y. Proposal for Highly Nonlinear Dispersion-Flattened Octagonal Photonic Crystal Fibers. IEEE Photonics Technology Letters. 2008; 20(4): 249-251.

[36] Kaijage SF, Ouyang Z, Jin X. Porous-Core Photonic Crystal Fiber for Low Loss Terahertz Wave Guiding. IEEE Photonics Technology Letters. 2013; 25(15): 1454-1457.

[37] Liu M, Yang J, Zhu T. Design of large-mode-area multi-core photonic crystal fibers with low confinement loss and dispersion. Jetp Letters. 2015; 102(5): 274-278.

[38] Ademgil H, Haxha S. Highly Birefringent Photonic Crystal Fibers With Ultralow Chromatic Dispersion and Low Confinement Losses. Journal of Lightwave Technology. 2008; 26(4): 441-448.

[39] Abdelaziz I, Abdelmalek F, Ademgil H. Enhanced Effective Area Photonic Crystal Fiber With Novel Air Hole Design. Journal of Lightwave Technology. 2010; 28(19): 2810-2817. 